Pensamiento Crítico Vol. 19 № 2, pp. 119-136

\title{
La administración de fondos privados de pensiones de Perú frente a las crisis financieras internacionales (1993-2013)
}

The administration of private pension funds in peru facing international

financial crises (1993: 2013)

Wilmer Flórez G *

"La ganancia actuarial o expectativa matemática de ganancia calculada de acuerdo con las posibilidades existentes -si es que puede calcularse, cosa que es dudosadebe ser suficiente para compensar el riesgo del desengaño" (Keynes, 2001:148).

\section{RESUMEN}

Los niveles de riesgo generados por las crisis financieras podrían afectar de manera sustancial el bienestar económico y social de las personas aportantes a un fondo de pensiones, ya sea este privado o público. El objetivo del presente artículo es el estudio de la administración de fondos privados de pensiones de Perú y la medición de su relación con las siete crisis financieras internacionales desarrolladas entre los años 1993 y 2013: crisis mexicana, asiática, rusa, brasileña, argentina, hipotecaria subprime y la crisis de Europa. Se realizó un estudio descriptivo correlacional para contrastar los vínculos de relación entre la rentabilidad de los fondos de

1 Magíster en Economía con mención Finanzas por la Universidad Nacional Mayor de San Marcos (UNMSM). Magíster en Administración Estrategia de Empresas por la Pontificia Universidad Católica del Perú (PUCP). Docente en la Universidad Nacional de San Antonio Abad del Cusco (UNSAAC). 


\section{Pensamiento Crítico Vol. 19. $\mathrm{N}^{\circ} 2$}

pensión de Perú y los índices bursátiles de las economías en crisis según cada caso, y su correspondiente temporalidad. Para determinar la eficiencia financiera de las inversiones de los fondos de pensiones peruanos fue necesario calcular el índice de Sharpe de los principales fondos de pensiones de Latinoamérica considerando horizontes temporales de cinco, diez y quince años, para mayor perspectiva. Se encontró que la administración de fondos de pensiones tuvo una correlación significativa con las crisis de Asia, Rusia, Brasil, Argentina, EE.UU. y la crisis de Europa, salvo la crisis mexicana. La crisis financiera con el mayor grado de asociación lineal (Estimado por el coeficiente de Pearson) y mayor impacto adverso fue la crisis hipotecaria subprime de Estados Unidos. Se encontró que los resultados de eficiencia financiera (según el índice de Sharpe) de los fondos de pensiones de Perú estuvieron por debajo del promedio de la región.

Palabras clave: Administración de fondos de pensiones, crisis financieras, riesgo de inversiones.

\section{ABSTRACT}

The levels of risk generated by the financial crisis could affect substantially the economic and social well-being of contributors to a pension fund people whether this private or public. The aim of this article is the study of the administration of private funds pensions of Peru and the measurement of its relationship with the seven international financial crises developed between 1993 and 2013: subprime mortgage Crisis, Mexican, Asian, Russian, Brazilian, Argentine, and Europe's crisis. A correlational descriptive study was conducted to test the bonds of relationship between the yield of Peru pension funds and stock indices of economies in crisis according to each case, and its corresponding temporality. To determine the financial efficiency of the Peruvian pension funds investments, it was necessary to calculate the index of Sharpe's main Latin America pension funds whereas horizons of five, ten and fifteen years, for greater perspective. It was found that administration of pension funds had a significant correlation with the crises in Asia, Russia, Brazil, Argentina, USA and the crisis of Europe, except the Mexican crisis. The financial crisis with the highest degree of linear Association (Dear by the Pearson coefficient) and greater adverse impact was the mortgage crisis subprime United States. It was found that the results of efficiency financial (according to the Sharpe index) of pension funds in Peru were below the average in the region.

Keywords: Pension funds, financial crisis, investment risk management. 


\section{Wilmer flórez García}

\section{Introducción}

El sistema privado de pensiones (SPP) es en lo fundamental un sistema de protección. En ese contexto, Hayek (1991: 364) dijo: "La previsión para la vejez y las consecuencias que se derivan de la misma constituye el sector donde la mayoría de los países han contraído responsabilidades más importantes y el que probablemente ha de crear los más serios problemas". En ese orden de ideas, a partir de agosto del año 1993, con la creación de los fondos privados de pensiones en Perú, los ahorros para la jubilación de millones de personas se encuentran expuestos, de manera intrínseca, al comportamiento favorable, así como adverso, de los mercados financieros y, en forma particular, de las crisis financieras internacionales. Entre los años 1993 y 2013 se han desarrollado siete crisis financieras internacionales: crisis mexicana (1995), asiática (1997), rusa (1998), brasileña (1999), argentina (2001), de las hipotecas subprime (2008) y europea (2010), las cuales han repercutido de forma sustancial en la administración y resultados de los fondos de pensiones de Perú. La normativa de inversiones instrumentada por la Superintendencia de Banca, Seguros y AFP (SBS) así como las estrategias de inversiones de las Administradoras de Fondos de Pensiones (AFP), no fueron inmunes a la caída de precios de los activos financieros del mercado, lo que derivó, en plena crisis del año 2008 , en una caída, en términos reales, de hasta un $-43 \%$ del portafolio del fondo tipo 3 y -23,9\% en el caso del fondo tipo 2. Conviene destacar las implicancias de las crisis financieras, como lo fuera en su momento para Argentina que en plena crisis hipotecaria, el año 2008, cerró su sistema privado de pensiones (SPP).

\section{Planteamiento del problema}

- ¿Cuál es el grado de relación entre las crisis financieras internacionales y la administración de los fondos privados de pensiones del Perú?

- ¿Cómo influye la regulación de inversiones del SPP en la administración de fondos privados de pensiones de Perú?

\section{Objetivos}

- Analizar el SPP y determinar el grado de influencia entre las crisis financieras y la administración de los fondos privados de pensiones de Perú. 


\section{Pensamiento Crítico Vol. 19. N²}

- Determinar la influencia de la regulación del sistema privado de pensiones sobre la administración de los fondos privados de pensiones.

\section{Justificación}

La disminución de los fondos de pensiones en tiempo de crisis ha originado profunda preocupación respecto la cobertura del riesgo de vejez de las 5.5 millones de personas afiliadas al sistema privado de pensiones (SPP) de Perú, lo cual también ocupa a los sistemas previsionales a nivel mundial. Según el diseño del SPP, las pensiones de jubilación representarán, en proporción, el 70\% de la remuneración en actividad (Asociación de AFP de Chile, 2006); no obstante, producto de las sucesivas crisis financieras internacionales, la rentabilidad de los fondos de pensiones ha registrado descensos significativos generando incertidumbre sobre la seguridad para garantizar pensiones al nivel esperado. De otro lado, destaca el elevado desconocimiento financiero de gran parte de los afiliados como para elegir el portafolio del tipo de fondo adecuado a su perfil, lo que podría acarrear decisiones inadecuadas que afecten de forma adversa al crecimiento de su fondo de pensiones para la jubilación. A ello se suma que durante los últimos diez años que terminan el 2013, la expectativa de vida de las personas mayores de 60 años se incrementó en 2.5 años alcanzando un promedio de 83 y 80 años para las mujeres y hombres respectivamente (Instituto Nacional de Estadística e Informática, 2013), manteniendo la tendencia de pago de pensiones por horizontes temporales mayores. Así las cosas, la medición empírica de la relación entre la administración de fondos de pensiones y las crisis financieras aporta con elementos de análisis objetivos sobre las influencias adversas de los desequilibrios financieros en el sistema a fin de generar propuestas legislativas que tiendan a salvaguardar los ahorros de jubilación para bienestar de las personas afiliadas. La racionalidad del funcionamiento del SPP toma forma a través del concepto de capitalización individual, de manera que, su principal objetivo es la preservación y el crecimiento de los ahorros para la jubilación de las personas afiliadas. En ese contexto, una disminución de los ahorros previsionales desequilibra la racionalidad del modelo por lo que la administración de los fondos debe buscar minimizar los impactos adversos generados por las crisis financieras internacionales. 


\section{Wilmer flórez García}

\section{Hipótesis}

- Las crisis financieras han impactado significativa y desfavorablemente en la administración de los fondos privados de pensiones de Perú.

- La regulación de inversiones del SPP influye positivamente en la administración de fondos privados de pensiones.

\section{Marco teórico}

\section{Teoría de agencia}

Jensen y Meckling (1976) definen como "agente" a una persona, conjunto de personas, directivos, de una organización, gestores de fondos, entre otros, que en virtud de un contrato formal actúan en representación de otra persona, agrupación de personas, accionistas, propietarios, consumidores, inversores, acreedores, etcétera, denominándolos "principales". La relación entre el agente y el principal implica un juego de intereses particulares, muchas veces divergentes entre sí, lo que podría afectar el proceso de toma de decisiones de los agentes. En esa línea, Fama y Jensen (1983: 2) afirmarían lo siguiente:

"Un factor importante en la supervivencia de las formas de organización es el control de los problemas de agencia. Estos problemas surgen porque los contratos no son escritos y son ejecutados sin costo. Los costes de agencia incluyen los costos de estructuración, supervisión, y la unión de una serie de contratos entre agentes con intereses en conflicto, además de la pérdida residual incurrida debido a que el costo de la plena vigencia de los contratos supera los beneficios".

La teoría de agencia describe la posibilidad de que los agentes actúen buscando intereses individuales en lugar de guiarse por los intereses de los principales, lo cual dará lugar a los llamados "problemas de agencia" (divergencias de intereses entre el agente y el principal), debido a la asimetría de información en favor del agente o en su defecto 


\section{Pensamiento Crítico Vol. 19. No 2}

porque los principales consideran más costoso tomar las medidas para que los agentes actúen como deben. Para que el agente subordine su interés personal al interés del principal, se tiene que asumir cierto costo implícito denominado por Jensen como costo de agencia con efectos tangibles como cualquier otro tipo de costo.

\section{Teoría de portafolio de Markowitz}

Los estudios de Markowitz (1952), producto de los cuales fuera galardonado con el premio Nobel en economía el año 1990, focalizan su análisis en la disminución del riesgo mediante la elección de activos con diferente comportamiento, es decir, con correlación negativa. De esta manera, la teoría de portafolios configura la combinación de diferentes alternativas de inversión generando un amplio espectro de retornos esperados para diferentes niveles de riesgos asociados a dichas inversiones. Es importante destacar que la valoración de dicho riesgo asociado a las inversiones y los retornos esperados son determinados por las siguientes identidades:

a) El riesgo asociado de la inversión tiene configuración matemática a través de la varianza ( $\delta 2)$ como medida de dispersión o su raíz cuadrada, es decir, la desviación típica $(\delta)$ de los retornos de aquellos instrumentos de inversión o la cartera, en un horizonte de tiempo determinado. Al respecto, la varianza del rendimiento de mercado es el valor esperado del cuadrado de las desviaciones respecto el rendimiento esperado (Brealey, Myers y Allen, 2006):

$$
\operatorname{Varianza}(\mathrm{m})=\text { valor esperado }(\mathrm{m}-\mathrm{rm}) 2
$$

En donde m es la rentabilidad realizada, y la rm viene a ser la rentabilidad esperada.

b) El retorno esperado, de un activo o cartera de inversión, es determinado por una medida de tendencia central, es decir, calculando el promedio ponderado de los rendimientos de la cartera en un horizonte de tiempo determinado:

$E\left(R_{c}\right)=W_{1} E\left(R_{1}\right)+W_{2} E\left(R_{2}\right)+\ldots+W_{n} E\left(R_{n}\right)$

En donde $\mathrm{E}(\mathrm{Ri})$ es el rendimiento esperado de la cartera o de los activos individuales y Wi, es la ponderación o peso asignado a cada activo de inversión. 


\section{Wilmer Flórez García}

La teoría de portafolio de Markowitz asume que la media de inversionistas del mercado financiero posee de manera natural cierta aversión al riesgo, de esta manera para configurar un portafolio de inversión se parte de tres hipótesis (Court, 2010: 216): (1) La rentabilidad de cualquier título o cartera es una variable aleatoria de carácter subjetivo, cuya distribución de probabilidad para el periodo de referencia es conocido por el inversor. El valor medio o esperanza matemática de dicha variable aleatoria se acepta como medida de la rentabilidad de la inversión, (2) Se acepta como medida de riesgo la dispersión, medida por la varianza o la desviación estándar, de la variable aleatoria que describe la rentabilidad, ya sea de un valor individual o de una cartera, y (3) La conducta del inversor le lleva a preferir aquellas carteras con una mayor rentabilidad y menor riesgo. De esta manera, tomando en cuenta las consideraciones de inversión descritas, la figura 1 muestra la selección de carteras eficientes (Brealey, Myers y Allen, 2006: 202). Al respecto, el interior de dicha figura muestra las posibles alternativas de inversión con un determinado nivel de rentabilidad y desviación típica, así cada diamante representa el rendimiento esperado y la desviación típica de una alternativa de inversión determinada. En línea, las carteras preferidas y seleccionadas son aquellas que presentan las más altas rentabilidades con un nivel de riesgo (desviación típica) menor, encontrándose que las carteras más eficientes (con mayor rentabilidad y menor riesgo) serían las carteras A, B, C y D respectivamente, ello debido a que tienen un desempeño superior, es decir, frente a un nivel de riesgo similar poseen una rentabilidad superior.

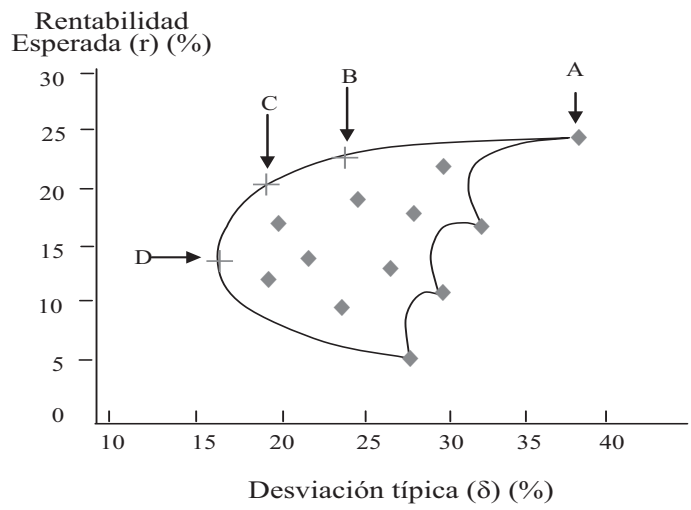

Figura 1. Frontera de carteras eficientes.

Fuente. Tomado de Principios de Finanzas Corporativas, por Brealey, Myers y Allen (2006). 


\section{Pensamiento Crítico Vol. 19. № 2}

\section{El índice de Sharpe}

Sharpe (1994) construye este índice tomando como referencia el modelo media varianza de Harry Markowtz. Uno de los supuestos de este índice es que los inversores con aversión al riesgo toman preocupación por la volatilidad global de sus inversiones, por lo tanto, les es más útil una evaluación de la eficiencia financiera tomando en cuenta la volatilidad global de los rendimientos, es decir, consideran tanto el riesgo sistemático como y el riesgo no sistemático del portafolio (del fondo pensionario para el presente estudio). En línea, para los inversores adversos al riesgo uno de los índices de eficiencia financiera más adecuados sería el índice de Sharpe, debido a que dicho ratio es un cociente que resulta de la división de la prima por riesgo del fondo pensionario entre la volatilidad total de los rendimientos del fondo, es decir, entre las desviación estándar de dichos rendimientos. El índice de Sharpe mide el rendimiento obtenido del portafolio por cada unidad de riesgo asumido. La formalización de este índice es la siguiente (Court y Tarradellas, 2010):

$$
\frac{S_{c}=E\left(R_{c}\right)-r_{f}}{\sigma_{c}}
$$

En donde, Sc, es el índice de Sharpe para el portafolio, E(Rc), la rentabilidad esperada del portafolio, rf, el rendimiento del activo libre de riesgo, y $\sigma \mathrm{c}$, es la desviación estándar de la rentabilidad del portafolio.

\section{Método}

Para determinar los vínculos relacionales a posteriori entre las variables cuantitativas del estudio se recurre al análisis estadístico y cuantificación correlacional. El coeficiente de correlación sirve de soporte para medir la dependencia lineal entre las variables de estudio, aspecto que se refuerza con lo sostenido por Gujarati (2004: 23): "En el análisis de correlación, el objetivo principal es medir la fuerza o grado de asociación lineal entre dos variables". La variable relevante de la administración de fondos de pensiones es el valor cuota, el cual es publicado por la Superintendencia de Banca, Seguros y AFP (SBS), así, para estudiar el grado de correlación entre el valor cuota y las crisis financieras se definieron

\section{I26}




\section{Wilmer Flórez García}

siete intervalos temporales de estudio, uno por cada una de las siete crisis estudiadas: crisis mexicana, crisis asiática, crisis rusa, crisis brasileña, crisis argentina, crisis de las hipotecas subprime y crisis europea. Se obtuvo data estadística de fuente secundaria de Yahoo Finance de las principales bolsas de valores de las economías en crisis, tomando como punto de inicio la fecha de estallido de cada crisis financiera hasta la fecha de término del deterioro de las acciones bursátiles correspondientes. Con base a esta data se procedió a construir series de datos con las variaciones diarias y mensuales según el caso (véase cuadro 1). Una vez construida las bases de datos, se procedió a contrastar la hipótesis mediante el uso del aplicativo Statistical Processor for Social Science (SPSS) para la obtención de tablas de referencia cruzada con el coeficiente de correlación lineal y la correspondiente prueba de validación de significancia al $0.01,0.05$ y 0.10 respectivamente.

\section{CUADRO 1}

Variables de la hipótesis 1

\begin{tabular}{cll}
\hline $\mathrm{N}^{\circ}$ & Variable & Frecuencia \\
\hline 1 & Valor Cuota Fondo 1 del SPP (01 Feb. 2006 - 31 Dic. 2013). & Diaria \\
2 & Valor Cuota Fondo 2 del SPP (02 Jul. 1997 - 31 Dic. 2013). & Diaria \\
3 & Valor Cuota Fondo 3 del SPP (01 Feb. 2006 - 31 Dic. 2013). & Diaria \\
4 & Valor Cuota Fondo 2 del SPP (Ago. 1993 - Dic. 2013). & Mensual \\
5 & Índice IPC de México (Set. 1994 - Feb. 1995). & Mensual \\
6 & Índice Hang Seng de Hong Kong (2 de Jul. 1997 - 8 Oct. 1998). & Diaria \\
7 & Índice RTSI de Rusia (17 de Ago. 1998 - 6 Oct. 1998). & Diaria \\
8 & Índice IBOVESPA de Brasil (14 de May. 1998 - 15 Ene. 1998). & Diaria \\
9 & Índice MERVAL de Argentina (03 de Mar. 2000 - 05 Dic. 2001). & Diaria \\
10 & Índice DJI de Estados Unidos (9 de Ago. 2007 - 9 Mar. 2009). & Diaria \\
11 & Índice IBEX de España (23 de Abr. 2010 - 31 Dic. 2013). & Diaria
\end{tabular}

Fuente. Elaboración del autor con base a datos de "Valor cuota diario" y "Valor cuota promedio mensual" por la Superintendencia de Banca, Seguros y AFP (SBS), 2014; y "Historical Prices" por Yahoo Finance. Recuperado de http://www.sbs.gob.pe y http://finance.yahoo.com.

Por otro lado, Marcel y Tapia (2010: 10) sostienen que "Las regulaciones sobre la inversión de los fondos de pensiones influyen no solo sobre su crecimiento de largo plazo, sino también sobre su volatilidad". En ese contexto, para confirmar o rechazar la 


\section{Pensamiento Crítico Vol. 19. $\mathrm{N}^{\circ} 2$}

Hipótesis 2 se realiza un análisis cuantitativo mediante el ratio Sharpe de los fondos de pensiones de Perú en comparación a los fondos de pensiones de la región: Colombia, Costa Rica, Chile, El Salvador, México y Uruguay, a fin de determinar las implicancias de la regulación del SPP sobre las inversiones y resultados de los fondos de pensiones de Perú. Para dicho fin, se consiguió data estadística de fuente secundaria de la Asociación Internacional de Organismos de Supervisión de Fondos de Pensiones (AIOS) que recoge data oficial de los diferentes organismos reguladores de los fondos de pensiones de la región (cuadro 2 ).

\section{CUADRO 2}

Variables de la hipótesis 2

\begin{tabular}{cll}
\hline N $^{\circ}$ & Variable & Frecuencia \\
\hline 1. & Rentabilidad Real Fondos de Pensión Colombia (1999-2013). & Anual \\
2. & Rentabilidad Real Fondos de Pensión Costa Rica (2002-2013). & Anual \\
3. & Rentabilidad Real Fondos de Pensión Chile (1999-2013). & Anual \\
4. & Rentabilidad Real Fondos de Pensión El Salvador (1999-2013). & Anual \\
5. & Rentabilidad Real Fondos de Pensión México (1999-2013). & Anual \\
6. & Rentabilidad Real Fondos de Pensión Uruguay (1999-2013). & Anual \\
7. & Rentabilidad Real Fondos de Pensión Perú (1999-2013). & Anual \\
\hline
\end{tabular}

Fuente. Elaboración del autor con base a "Boletín estadístico AIOS: 1999 a 2009" y "Estadísticas periódicas: 2010 a 2012" por la Asociación Internacional de Organismos de Supervisión de Fondos de Pensiones (AIOS), 2014. Recuperado de http://www.aiosfp.org/estadisticas/boletin-estadistico-aios/

\section{Resultados}

La duración del estrés financiero puede alcanzar días, semanas, meses o inclusive años (Kindleberger, 2012). De esta manera, durante los 20 años de vida del sistema privado de pensiones (SPP), los fondos pensionarios fueron impactados, en mayor o menor medida, por siete crisis financieras internacionales con duraciones e intensidad sumamente heterogénea. De esta manera, tal como se aprecia en la figura 2, dichas crisis financieras se sucedieron en la siguiente secuencia: (1) la crisis mexicana, que implicó el deterioro de su índice bursátil, el IPC, entre setiembre de 1994 y febrero 1995, (2) la crisis asiática que se inició en Tailandia el mes de julio del año 1997, extendiendo 
su impacto hasta octubre de 1998, (3) la crisis rusa que estalló en agosto de 1998, generando la caída del índice bursátil ruso, el RTSI, hasta el 6 de octubre del mismo año, (4) la crisis brasileña, entre mayo de 1998 y enero de 1999, la cual por efecto contagio influyó en (5) la crisis argentina cuyo desencadenamiento recae hacia los años 2000 y 2001, (6) La crisis de las hipotecas subprime de Estados Unidos que inicia el 9 de agosto del 2007 hasta marzo del 2009 y finalmente (7) La crisis financiera europea que viene golpeando los mercados financieros desde abril del año 2010.

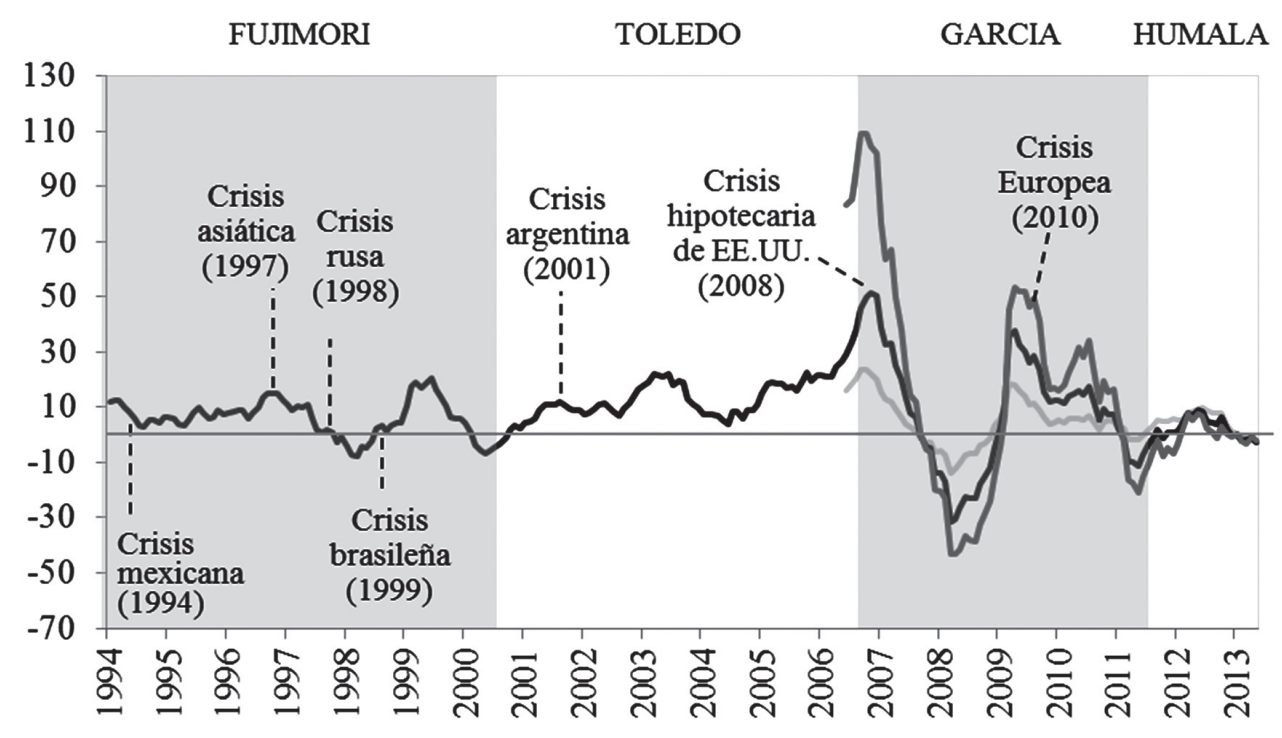

Rentab. Real Fondo 1 Rentab. Real Fondo 2 Rentab. Real Fondo 3

Figura 2. Crisis financieras y rentabilidad real del SPP por tipo de fondo.

Fuente. Elaboración del autor con base a "Rentabilidad real" a 12 meses por la Superintendencia de Banca, Seguros y AFP (SBS), 2014. Recuperado de http://www.sbs.gob.pe/app/stats/EstadisticaBoletinEstadistico. asp? $=31 \#$

Se aprecia que durante los periodos de 1998, 2000, 2008, 2011 y 2013 (SBS, 2014), los fondos de pensiones registraron rendimientos negativos respecto a sus inversiones. Los mecanismos de transmisión de las crisis económicas hacia los mercados financieros y en particular hacia los fondos de pensiones se canalizan en primer término 


\section{Pensamiento Crítico Vol. 19. № 2}

a través de los mercados bursátiles donde se realiza la transferencia de recursos para financiamiento e inversión (acciones y bonos). De esta manera, debido a su estructura de inversiones, el tipo de fondo más expuesto a los vaivenes de las crisis financieras es el Fondo Tipo 3 (Fondo de apreciación de capital) toda vez que su portafolio considera un $80 \%$ de colocaciones en activos de renta variable (acciones) y la diferencia, es decir el $20 \%$, se invierte en instrumentos de renta fija (bonos). En el caso de los fondos tipo 2 y 1 , la inversión en acciones representa el $45 \%$ y $10 \%$ del portafolio de inversiones respectivamente.

En tiempos de crisis, el primer impacto se da en la pérdida de confianza de los inversionistas en la economía lo que deriva en una devaluación de la moneda, el incremento de los tipos de interés y posterior endeudamiento estatal. Así, estos fenómenos financieros suelen generar un pánico financiero afectando a los activos cotizados en las bolsas de valores donde se cotizan las inversiones de los fondos de pensiones. De esta manera, el cuadro 3 recoge los resultados de la correlación Pearson entre las variables de estudio. Se aprecia que la crisis financiera de mayor correlación con los fondos privados de pensiones de Perú fue la crisis hipotecaria subprime con coeficientes de correlación Pearson de 0,575 y 0,558 para el caso de los fondos tipo 3 y 2 y el índice Dow Jones respectivamente. Este nivel de correlación se reflejó en la caída de rentabilidad de dichos fondos durante el año 2008: - 41,7 y - $26,74 \%$ en cada caso. En el otro extremo se aprecia el bajo nivel de correlación con la crisis mexicana que alcanzó un nivel de correlación Pearson de 0,14, no obstante, este resultado no tolera el nivel de significancia de $0.01,0.05$ y 0.10 requeridos. En el caso de la correlación con los índices bursátiles argentino y asiático (Se toma como referencia el índice Hang Seng de Hong Kong) se aprecia niveles de correlación relativamente mayores: 0,270 y 0,355 respectivamente. Por otro lado, se observa que, el nivel de correlación lineal entre el RTSI de Rusia y el Fondo 2 del SPP alcanzó un nivel de 0,288, no obstante, el nivel de significancia fue de 0,084. En esa línea de análisis, la correlación entre el índice de referencia de Europa para el estudio: el IBEX de España y los fondos de pensiones presenta una correlación relativamente elevada: $(0,487,0,393$ y 0,485 para los fondos tipo 1 , tipo 2 y tipo 3 respectivamente). 


\section{Wilmer flórez García}

\section{CUADRO 3}

Correlaciones de Pearson entre los índices bursátiles de los países en crisis y los fondos tipo 1, 2 y 3 del SPP

\begin{tabular}{|c|c|c|c|c|}
\hline & & $\begin{array}{l}\text { Fondo Tipo } 2 \\
\text { del SPP }\end{array}$ & $\begin{array}{l}\text { Fondo Tipo } 1 \\
\text { del SPP }\end{array}$ & $\begin{array}{l}\text { Fondo Tipo } 3 \\
\text { del SPP }\end{array}$ \\
\hline Índice de Precios y & Correlación de Pearson & 0,14 & & \\
\hline \multirow{2}{*}{$\begin{array}{l}\text { Cotizaciones (IPC)- } \\
\text { México }\end{array}$} & Sig. (bilateral) & 0,525 & & \\
\hline & $\mathrm{N}$ & 23 & & \\
\hline \multirow{3}{*}{$\begin{array}{l}\text { Índice Hang Seng - } \\
\text { Hong Kong }\end{array}$} & Correlación de Pearson &, $355^{* *}$ & & \\
\hline & Sig. (bilateral) &, 000 & & \\
\hline & $\mathrm{N}$ & 330 & & \\
\hline \multirow[t]{3}{*}{ Índice RTSI - Rusia } & Correlación de Pearson & 0,288 & & \\
\hline & Sig. (bilateral) & 0,084 & & \\
\hline & $\mathrm{N}$ & 37 & & \\
\hline \multirow{3}{*}{$\begin{array}{l}\text { Índice IBOVESPA } \\
\text { - Brasil }\end{array}$} & Correlación de Pearson &, $315 * *$ & & \\
\hline & Sig. (bilateral) & ,000 & & \\
\hline & $\mathrm{N}$ & 263 & & \\
\hline \multirow{3}{*}{$\begin{array}{l}\text { Índice MERVAL - } \\
\text { Argentina }\end{array}$} & Correlación de Pearson &, $270 * *$ & & \\
\hline & Sig. (bilateral) &, 000 & & \\
\hline & $\mathrm{N}$ & 452 & & \\
\hline \multirow{3}{*}{$\begin{array}{l}\text { Índice Dow Jones } \\
\text { Industrial de } \\
\text { EE.UU. }\end{array}$} & Correlación de Pearson &, $317 * *$ &, $558 * *$ &, $575^{* *}$ \\
\hline & Sig. (bilateral) &, 000 &, 000 &, 000 \\
\hline & $\mathrm{N}$ & 408 & 408 & 408 \\
\hline \multirow{3}{*}{$\begin{array}{l}\text { Índice IBEX de } \\
\text { España }\end{array}$} & Correlación de Pearson &, $393^{* *}$ & $487 * *$ &, $485^{* *}$ \\
\hline & Sig. (bilateral) &, 000 &, 000 &, 000 \\
\hline & $\mathrm{N}$ & 957 & 957 & 957 \\
\hline
\end{tabular}

**. La correlación es significativa en el nivel 0,01 (2 colas).

Por otro lado, con relación a la eficiencia de la regulación del sistema privado de pensiones, el cuadro 4 muestra que la rentabilidad de los fondos de pensiones peruanos es superior al promedio de la región; no obstante, la volatilidad estimada por la desviación estándar (riesgo) presenta niveles significativamente superiores al resto de 
fondos de pensiones de la región para los periodos evaluados: cinco, diez y quince años. Considerando un horizonte de evaluación de cinco años, entre los años 2009 y 2013, los fondos de pensiones con mayor eficiencia de inversiones, según el ìndice Sharpe, corresponden a los fondos de pensiones de Costa Rica y México con coeficientes de 1.9 y 1.4 respectivamente, mientras que los fondos de pensiones de Perú obtuvieron el más bajo desempeño alcanzando un índice Sharpe de 0.5. Por otro lado, considerando un horizonte de evaluación a diez y quince años, los fondos de pensiones de Perú presentaron uno de los ratios de eficiencia más bajos: 0.5 y 0.6 para dichos períodos.

\section{CUADRO 4}

Eficiencia de inversiones de los fondos de pensiones de América Latina (1999-2013)

\begin{tabular}{|c|c|c|c|c|c|c|c|c|}
\hline & Período & Chile & Colombia & $\begin{array}{l}\text { Costa } \\
\text { Rica }\end{array}$ & $\begin{array}{c}\text { El } \\
\text { Salvador }\end{array}$ & México & Perú & Uruguay \\
\hline & 1999 & 16,3 & 11,7 &.- & 14,1 & 13,1 & 18,7 & 10,9 \\
\hline & 2000 & 4,4 & 7,8 &.- & 7,9 & 7,2 & $-6,7$ & 7,1 \\
\hline & 2001 & 6,7 & 10,5 &.- & 7,7 & 6,1 & 11,1 & 5,5 \\
\hline & 2002 & 3,0 & 9,7 & 7,1 & 2,4 & 4,7 & 11,2 & 40,6 \\
\hline & 2003 & 10,5 & 9,4 & 9,8 & 4,8 & 6,2 & 21,2 & 27,6 \\
\hline & 2004 & 8,9 & 10,4 & 2,5 & 2,3 & 4,5 & 5,6 & 6,6 \\
\hline & 2005 & 4,6 & 19,0 & 4,1 & 1,5 & 8,0 & 18,4 & 4,6 \\
\hline & 2006 & 15,8 & 2,7 & 10,3 & 1,2 & 8,7 & 26,8 & 9,5 \\
\hline & 2007 & 5,0 & 0,9 & $-0,7$ & 1,4 & 2,5 & 20,2 & 0,5 \\
\hline & 2008 & $-18,9$ & $-2,7$ & $-9,0$ & $-2,3$ & $-6,5$ & $-26,7$ & $-21,5$ \\
\hline & 2009 & 22,5 & 26,8 & 9,2 & 5,6 & 10,2 & 32,9 & 30,0 \\
\hline & 2010 & 9,3 & 15,0 & 2,2 & 2,4 & 9,0 & 16,0 & 14,4 \\
\hline & 2011 & $-3,8$ & $-4,0$ & 4,1 & $-2,1$ & 3,1 & $-11,3$ & 3,7 \\
\hline & 2012 & 4,6 & 13,2 & 5,0 & 4,4 & 11,7 & 8,9 & 4,8 \\
\hline & 2013 & 4,7 & $-0,5$ & 8,9 & 1,5 & 0,2 & $-2,8$ & $-1,7$ \\
\hline 5 años & Media & 7,5 & 10,1 & 5,9 & 2,4 & 6,8 & 8,7 & 10,3 \\
\hline (2009: & D. Estándar & 9,7 & 12,5 & 3,1 & 3,0 & 5,0 & 17,1 & 12,5 \\
\hline 2013) & Ind. Sharpe & 0,8 & 0,8 & 1,9 & 0,8 & 1,4 & 0,5 & 0,8 \\
\hline
\end{tabular}


Wilmer flórez García

\begin{tabular}{llccccccc}
10 años & Media & 5,3 & 8,1 & 3,7 & 1,6 & 5,1 & 8,8 & 5,1 \\
(2004: & D. Estándar & 11,1 & 10,4 & 5,7 & 2,5 & 5,5 & 18,2 & 12,9 \\
2013) & Ind. Sharpe & 0,5 & 0,8 & 0,6 & 0,6 & 0,9 & 0,5 & 0,4 \\
15 años & Media & 6,2 & 8,7 & 4,5 & 3,5 & 5,9 & 9,6 & 9,5 \\
(1999: & D. Estándar & 9,4 & 8,4 & 5,5 & 4,2 & 4,9 & 15,8 & 14,7 \\
2013) & Ind. Sharpe & 0,7 & 1,0 & 0,8 & 0,8 & 1,2 & 0,6 & 0,6 \\
\hline
\end{tabular}

Fuente. Elaboración del autor con base a "Boletín estadístico AIOS: 1999 a 2009" y "Estadísticas periódicas: 2010 a 2013" por la Asociación Internacional de Organismos de Supervisión de Fondos de Pensiones (AIOS), 2014; Recuperado de: http://www.aiosfp.org/estadisticas/boletin-estadistico-aios/

De acuerdo a la teoría financiera, el índice de Sharpe permite calcular el rendimiento o ganancia obtenida por unidad de riesgo asumido. De esta manera, si bien los rendimientos de los fondos de pensiones de Perú fueron los más altos a nivel regional, ello implicó una mayor volatilidad, es decir, a costa de un mayor nivel de riesgo. Estos resultados se explican en buena medida debido a las características del mercado bursátil local. En esa línea, al 2 de enero del 2014, el 48.2\% de acciones integrantes del Índice General de la Bolsa de Valores de Lima (IGBVL), es decir 14 de 29 acciones, se encontraban relacionadas al sector minero. Un elemento adicional a considerar es el reducido nivel de liquidez y profundidad del mercado bursátil local, de manera que, ante situaciones de estrés financiero, la gestión de riesgos por parte de los inversionistas conlleva serias limitaciones para liquidar o tomar posiciones de inversión.

\section{Conclusiones}

1. Los resultados de la administración de los fondos de pensiones presentan una correlación lineal con las crisis financieras. El estudio confirmó la hipótesis general, referida a la correlación lineal entre la administración de fondos privados de pensiones (expresada en el valor cuota) con seis de las siete crisis financieras internacionales estudiadas: crisis asiática, rusa, brasileña, argentina, de las hipotecas subprime y europea. La crisis financiera con el mayor grado de asociación lineal y mayor impacto adverso fue la crisis hipotecaria subprime con coeficientes de correlación lineal Pearson de 0.487, 0.393 y 0.485 con los portafolios de los fondos tipo 1,2 y 3 , respectivamente. Así, durante los 19 meses de deterioro del índice bursátil Dow Jones, contados a partir de agosto del 2007, los valores cuota promedio de los 


\section{Pensamiento Crítico Vol. 19. No 2}

fondos de pensiones de Perú se deterioraron en niveles de $-1.2 \%$ para el portafolio del fondo tipo $1 ;-23.9 \%$ para el portafolio del fondo tipo 2 y $-43 \%$ para el portafolio del fondo tipo 3. Las crisis financieras tuvieron implicancias en el sistema privado de pensiones (SPP) a través de cuatro mecanismos de transmisión: (1) el 57\% del total de pensionistas del sistema cobran sus pensiones en dólares, lo que implica la elevada exposición al riesgo cambiario; entre los años 2002 y 2013 el dólar registro una depreciación del $20 \%,(2)$ a través de la reducción de las tasas de interés técnico utilizadas en el cálculo de pensiones, derivando en una reducción de los montos de las pensiones, (3) por medio de la disminución del rendimiento patrimonial (ROE) tanto de las Administradoras de Fondos de Pensiones como para las empresas aseguradoras, que ofrecen rentas vitalicias, que el año 2008 registraron niveles negativos de rendimiento: $-1.6 \%$ y $-1 \%$ respectivamente, y (4) por la reducción de los resultados de las inversiones de los fondos de pensiones, así como los niveles de cobertura de obligaciones técnicas de las empresas aseguradoras.

2. La regulación de inversiones del sistema privado de pensiones (SPP) no influye positivamente en los resultados de rentabilidad ajustada por riesgo de los fondos de pensiones del Perú (con relación al desempeño de los fondos de pensiones de la región). La evidencia empírica del estudio muestra que la rentabilidad ajustada por riesgo a mediano y largo plazo (cinco, diez y quince años) de los fondos de pensiones de Perú estuvo por debajo del promedio de la región, ello se explicaría principalmente por la elevada volatilidad de los fondos de pensiones peruanos frente a los fondos de pensiones de la región. Así las cosas, la normativa de inversiones del SPP requiere un mayor nivel de estudio y desarrollo de alternativas que haga más eficiente la gestión de riesgos de los fondos de pensiones, en especial en coyunturas de crisis financieras. Actualmente, la normativa del SPP establece que un afiliado puede elegir el fondo tipo 3 (el fondo de mayor volatilidad del SPP peruano) hasta los 59 años de edad, exponiendo innecesariamente sus ahorros jubilatorios ante las crisis y situaciones de estrés financiero. En esa línea de análisis, para una elección "informada" de portafolio de inversión (1,2 o 3) por parte de los afiliados, se recomienda normar la implementación de un sistema de incentivos e indicadores para que las Administradoras de Fondos de Pensiones (AFP) desarrollen programas informativos específicos en la elección de tipo de fondo. 


\section{Wilmer flórez García}

\section{Referencias bibliográficas}

Asociación Internacional de Organismos de Supervisión de Fondos de Pensiones (AIOS). 2014. 12 de mayo 2014. Disponible en http://www.aiosfp.org

Asociación de AFP de Chile (2006). Sistema de AFP: Mitos y realidades. Departamento de estudios de la Asociación de AFP de Chile. 20 de marzo 2014. Disponible en http://www.fiap.cl/prontus_fiap/site/artic/20130306/asocfile/20130306131501/ mitos_y_realidades_sistema_afp_de_chile_marzo_2006_pdf

Brealey R., Myers S. y Allen F. (2006). Principios de Finanzas Corporativas. Madrid, España: McGraw Hill.

Court, Eduardo (2010). Finanzas Corporativas. Buenos Aires, Argentina: Cengage Learning.

Fama, E. y Jensen, M. (1983). Agency problems and residual claims. Journal of Law \& Economics, Vol XXVI (June 1983). 15 de marzo del 2014. Disponible en http:// papers.ssrn.com/sol3/papers.cfm?abstract_id $=94032$

Gujarati, Damodar (2004). Econometría. México D.F., México: McGraw-Hill.

Hayek, Friedrich (1991). Los fundamentos de la libertad. Madrid, España: Union Editorial S.A.

Instituto Nacional de Estadística e Informática [INEI] (2013). Nota de prensa: Dos millones 807 mil personas en nuestro país tienen 60 y más años de edad. 18 de Mayo del 2014. Disponible en http://m.inei.gob.pe/media/MenuRecursivo/ noticias/23082013c.pdf

Jensen, M. y Meckling, W. (1976). Theory of the Firm: Managerial Behavior, Agency Costs and Ownership Structure. Journal of Financial Economics (JFE), Vol. 3, No. 4, 1976. P.5. 16 de Mayo del 2014. Disponible en http://papers.ssrn.com/sol3/papers. cfm?abstract_id $=94043$ 


\section{Pensamiento Crítico Vol. 19. № 2}

Keynes, John (2001). Teoría General de la Ocupación, el Interés y el Dinero (3 ${ }^{\mathrm{a}}$ ed.). México D.F., México: Fondo de Cultura Económica de Argentina S.A.

Kindleberger, Charles (2012). Manías, pánicos y cracs: Historia de las crisis financieras. Barcelona, España: Editorial Planeta.

Marcel, M. y Tapia, W. (2010). Efectos de la crisis financiera sobre las pensiones en América Latina. Washington DC., Estados Unidos: working paper series; 152, Banco Interamericano de Desarrollo. 10 de Abril del 2014. Disponible en http://www10. iadb.org/intal/intalcdi/PE/2011/07613.pdf

Márkowitz Harry (1952). Portfolio selection. The Journal of Finance, Vol. 7, No. 1 (Mar., 1952), p. 77-91. 13 de Octubre del 2013. Disponible en http://www.jstor.org/discove $\mathrm{r} / 10.2307 / 2975974$ ?uid $=2 \&$ uid $=4 \&$ sid $=21102672956991$

Sharpe, William (1994). The Sharpe Ratio, en The Journal of Portfolio Management, Stanford University. 19 de abril de 2014. Disponible en http://www.stanford. edu/ wfsharpe/art/sr/sr.htm

Superintendencia de Banca, Seguros y AFP [SBS] (2014). Boletín Estadístico de AFP (Mensual). 10 de Junio del 2014. Disponible en http://www.sbs.gob.pe/app/stats/ EstadisticaBoletinEstadistico.asp? $\mathrm{p}=31$ 